\title{
HUGE MIMO TECHNOLOGY ROLE RECOGNITION FOR ITS EFFECTIVE 5G AND SATELLITE CONNECTIVITY IMPLEMENTATION
}

\author{
Dr B Suneela, Associate Professor, \\ Department of ECE, \\ Lords Institute of Engineering and Technology, Hyderabad, Telangana. \\ suneela@lords.ac.in
}

\begin{abstract}
Wireless networking provides major problems due to the large number of users (QoS). This level of demand is supposed to be satisfied by 5G technology. And with respect to satellite communication, there is also a broad data requirement. Because of the LOS requirements, as well as an increased number of cells, today's mobile phone technology can no longer meet this bandwidth an obvious solution to this problem would be using milli-metric wave communication (MIMO). It is important to use multiple antennas (MIMO) and space multiplex when using the MIM approach to improve the spectrum efficiency and data reliability. With MIM support beam-forming and channel information, the device is able to boost energy efficiency. Similar ideas can be applied to other technologies such as satellite communication. These researchers have successfully completed the tasks related to MIM, and have now proposed a prototype for the assessment.
\end{abstract}

Key words: 5G, Energy Efficiency (EE), Spectral Efficiency (SE), Channel State Information (CSI), Quality of Service (QoS) and Multiple Input Multiple Output (MIMO).

\section{I.INTRODUCTION}

Mobile telephony has become an important part of our everyday life. There are many applications for large numbers of devices these days, thanks to technology, but one drawback is that we are only limited to our creativity. Vast quantities of radio spectrum are used for different uses and/users. The mass adoption of
Internet of Things (IoT) is pushing businesses to satisfy the need for connecting everybody, person to person and everything to everything. All present systems have a unique features and functional needs in order to fulfill the requirements of the age we live in. $2 \mathrm{G}$ followed by $4 \mathrm{G}$, and is currently jumping to 5G. In cases where the line has strong stationary (connectivity) or no- cord (mobile) conditions, the $5 \mathrm{G}$ speeds will be up to $10 \mathrm{Gpbs}$; in more mobile (connectivity) and variable conditions, $1 \mathrm{G}$ will provide Internet with a peak rate of $1 \mathrm{Gbps}$. A broad bandwidth is also over the last few years; MIM is an emerging technology for future $5 \mathrm{G}$ and is the core of it. It has comes with high quality requirements for imagingand such.

It is needed to provide high spectral performance, mass device connectivity, lower latency, improved data rate, QoS, and lower cost. Academic research suggests new strategies to combat the challenges listed above, and industry has begun to introduce a few of them Speculation has it that millimeter-wave technology (mm Wave) would allow for greater bandwidth The primary advantages of MIMO include: they enable the acquisition of customers and a reduction in cost per customer acquisition. With these ultra-dense networks, it is anticipated that wireless throughput and power usage can also be improved by using more small cells [which means more of them] than traditional networks. An extensive transmitter and receiver range and the ability to achieve high data rate from just a handful 
of antennas. Additive noise and complicated digital effects can be reduced by using multiple receiving antennas [8-12] when two or more user equipment positions are in the same cell, spatial streams can be steered to a large number of User Equipment (UE) at the same time, at the same bandwidth. Some elements of $5 \mathrm{G}$ are being tested at various speeds. More research on the quality of Massive IN and Small Cells (MC) has been done in [than energy saving Massive MIM has been shown to be more energy efficient (as shown in cellular mobile phones) with a limited number of SC than when SC numbers are large. The solution can be evolved by converging on Machine-to-Internet-of-of-Things (M2I), small cells, and device-to-to-device (D2D) communications, which is demonstrated in Fig. 1 [14].

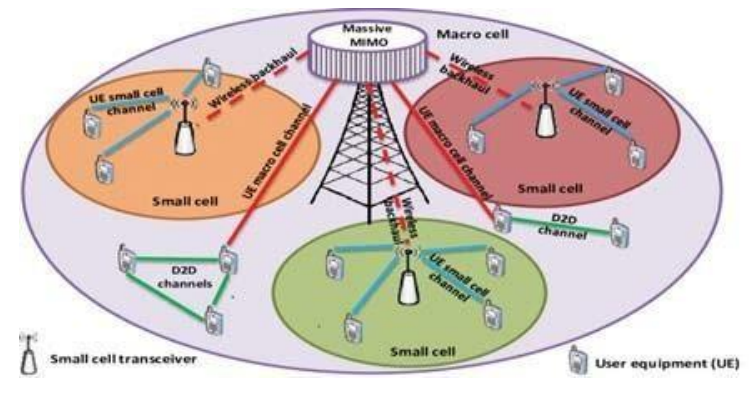

Fig.1.HetNet

As the operation becomes more complicated, HNet becomes a maze of imaginative uses. MIMO would be absolutely important in order to make useful spectral information and energy efficient. In Massive MIM, the implementation has too many avenues to follow. Channel performance also includes low-cost antennas, hardware loss compensation, characterization, and pilot errors this would alsoincrease the total data transmission rate. This paper defines MIM in relation to the cellular network in this report. Section III explains how MIM intends to address various software and design issues in development, with technology improvement in mind. At the end of the study, a number of tasks for $5 \mathrm{G}$ and satellite connectivity are suggested, which closes with an outline of a MIM design Thus the paper is laid out: In section II and section III, the setting and capabilities of the MIM are defined. Section IV contains the technological developments, and Section V presents the design problems. Finally, in Section VI, the applications are presented in Section VII.

\section{BACKGROUND}

\section{A. Cellular vs. Massive MIMO}

One has numbers (UE). The cellular network (comprises several BTS) and consumer equipment (counts devices) (UEs). Every UE will be linked to a BS to ensure service is available. The link from the link to BS is downlink (UL) (UL). A contemporary/ future cellular networks area rate in bit/s-kilometer per second. Bandwidth is the primary; D is average,

\section{Area throughput $\left[\mathrm{bit} / \mathrm{s} / \mathrm{km}^{2}\right]=$ $\mathrm{B}[\mathrm{Hz}] * \mathrm{D}\left[\right.$ cells $\left./ \mathrm{km}^{2}\right] * \mathrm{SE}[\mathrm{bit} / \mathrm{s} / \mathrm{Hz} / \mathrm{cell}]$} while energy is secondary. Area throughput is managed bythree variables that are independent of one another: B, D, and SE. Cell density and bandwidth specifications are all dictated by propagation conditions. One way to increase the throughput of cellular networks is to provide better coverage.

a. More band width allocation.

b. Deploy more BS sto increase network density

c. Increase SE of each cell.

Also important are basic specifications, such as increasing spectral efficiency, or energy efficiency, and improved hardware. Finally, the connection should be of a higher data rate and meet Quality of Service (QoS) requirements. CURRENT cellular technology is hindered by the problems like base station density and by things like buildings or tall structures that. These issues can be overcome by employing a broadband technology such as MIM 
with high-quality of service technology.

\section{B. Definition of Massive MIMO}

It operates on SD, improving overall system benefit and providing separate service to multiple users at the same time to improve throughput. Cell interference is more than BS antennas are used for effective suppression if the use of Ues increases so does the upload bandwidth. As long as we use TDD channels and multiple antennas, CSI acquisition overhead is negligible.

\section{BASICS OF MIMO:}

Also referred to as MIMO or L2 is a System of Huge Implementations, Large-Scale Antenna Systems, or Hyper MIM It is the most promising technology for providing data rates that are high enough to be needed. Most base stations have many large antennas which are dynamically arranged to better serve users who have one or more antennae. this limits contact in a bounded space Massive MIMO antenna has become practical because of the advancement of milli metric communication [19] It permits the installation of a great number of antennas in a tiny room. It is represented in figure 2 . TX and Rx both have multiple antennas are seen to have multiplexes. Each station may be transmitting the same frequency, and also have a different fading coefficients. This describes that LOS is not being needed for cellular. It does quite a bit to localize and diversify the spatial layers. The benefit of spatial multiplex is high capacity and quality of service.

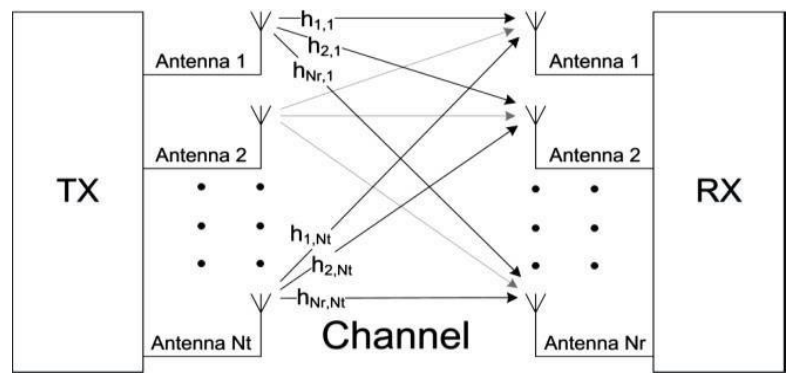

Fig.2.NxNMIMOBlockdiagram
System can be represented below.

$\mathrm{Y}_{\mathrm{Nr}}=\mathrm{H}_{[\mathrm{N}, \mathrm{N}]} \mathrm{X}_{\mathrm{Nt}}+\mathrm{Wn}$

For a simple $2 \times 2 \mathrm{MIMO}$,

$$
\begin{aligned}
& Y_{1}=H_{[1,1]} X_{1}+H_{[1,2]} X_{2} \\
& Y_{2}=H_{[2,1]} X_{1}+H_{[2,2]} X_{2}
\end{aligned}
$$

For this purpose, the receiver has a higher $\mathrm{S} / \mathrm{N}$ and a wider bandwidth; it results in higher data rates though at the same quality level. Fig. 3: All you need to do is implement this in hardware and it will be right.

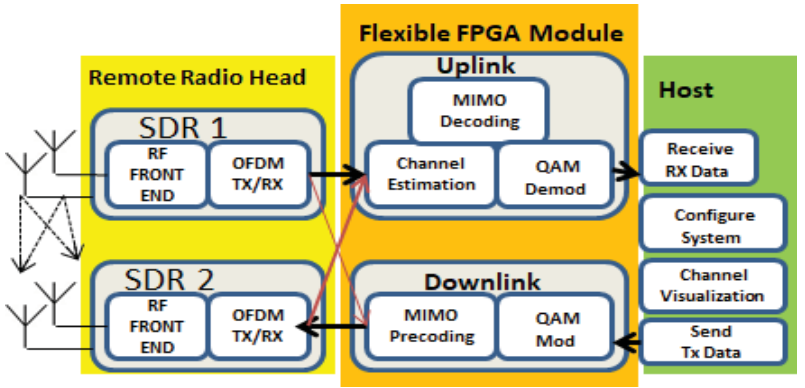

Fig.3.Hardware setup of Massive MIMO

We give Software Specified Radio (SDR) for IF and RF combinations to provide more flexibility for your choices. In addition to this MIMO configuration, the antenna has a "receive" and "transmit" mode later sections describe other aspects, which includes satellite communication, in additional subsections.

\section{A. MIMO Antenna}

Many-array antennas are constructed with numbers, proximity, and modes of operation in mind. Each of the patterns is a factor in the array's efficiency. The antenna shown in Fig. 4 is a simple planar. Highdirectivity can be given, but large size can result.

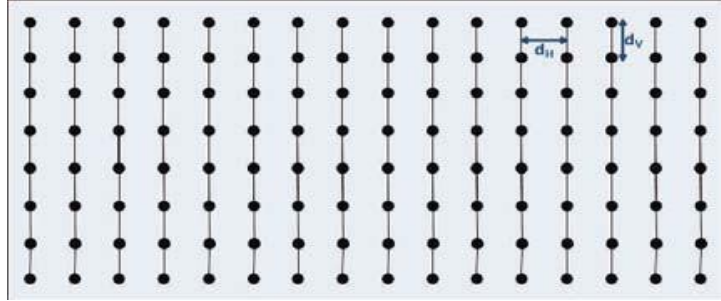

Fig.4. Plan array. 
There are a wide range of Antenna configurations for $5 \mathrm{G}$; however, many of them may be original and different Both rectilinear and rotund configurations can be used as well. Design decisions are dependent on the number of components, width of beams, strength of coupling, and compatibility. But it should be noted that in addition, considerations also include coverage, signal power, and channel capacity. Mostly in the 6, 27, and 60-70 GHz are used in the operation of this band. Several kinds of antennae including dipoles, conical, cylindrical, and written anten nas are under consideration.

\section{B. Beam forming Antenna:}

Additional antenna elements with phase differences result in a specific beam. To do more than one station, it can have several beams.

\section{MIMO for Backhaul Communication}

Conference between two cellular telephone towers (CBs) is known as backhauling up fibre, infrared, satellite connections and other back-up communications systems When a MIMO with a known position is employed, MIM will guide extremely focused beams between BSs.

\section{Massive MIMO for User Access}

Beam-forming techniques are available by using several antennas of varying sizes. Because of highwavelength directive beams and low side-lobe levels, interference-free communication is feasible. Telecommunications networks are now are generally provisioned for one device at a time; however, 5G systems would be able to handle many devices simultaneously. This application can be further improved using multiple antennas in smart phones and other portable devices number of antennas at both BSs and mobile devices.

\section{A. MIMO for Satellite Application.}

Assume that more than one satellite connected to a MIM encoder exist at distance $\mathrm{T}$ transmit information to more than one antenna over a time period dT The same information transmitted from both antenna 1 and antenna 2 is obtained by satellite $\mathrm{S} 1$, antenna $\mathrm{S} 2$ is divided by distance (d). When information is sent to a MIMdecoder, several MIM decoders will collect it. Spatial multiplexing is employed to boost bandwidth and priorities quality.

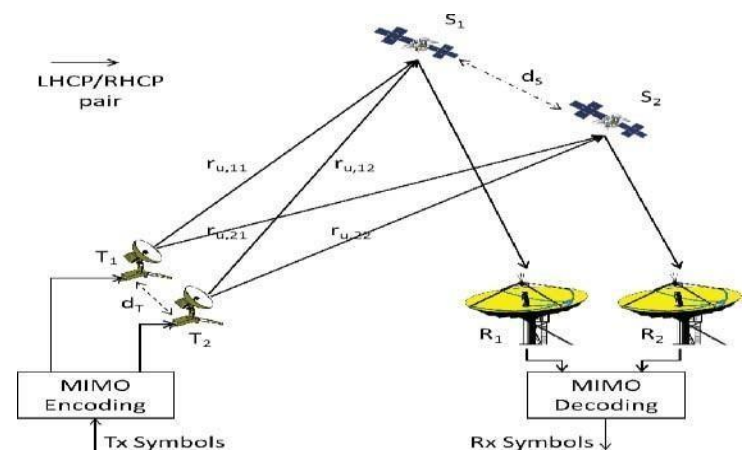

Fig.5.MIMO for satellite application

A material knowledge framework (MIM) is determined by changes to be applied to one or more technologies.

a) The Time Division Duplex uplink and downlink function can be performed in separate frequency blocks at the same time.

b) Getting high data rate and better quality of service are related to spatial multiplexing.

C) Benefit and greater directional precision be operated at the same time with numbers of beams of equal to or different.

\section{DESIGN CHALLENGES}

a. (Between BS and UE) Accurate channel state information (CSI) this is excellent for harvesting power.

b. An application which works well with dynamic beam steering for users that are on their feet and keeps beam overlap to a minimum in the event multiple users are working in one location.

c. Keep the radiation levels at a low, and break the radioactive sector. 


\section{APPLICATIONS}

Wireless internet connectivity is used in several non-medical, non-industrial settings, machine to machine-to-to-machine environments. It's important to use sensors, but they consume a lot of bandwidth. As in vehicle network: It may also be compared to a vehicle-to-to-to-vehicle connectivity programme, but more specifically it would be compared to an autonomous vehicle modern agriculture necessitates data about crop management, water management, and livestock management To get information on passenger trains, goods trains, goods, and the tracks the $5 \mathrm{G}$ network, all we need to do is register it. During calamities including flooding and cyclones, various individuals are stranded in regions of the world that are previously unknown. In the other side, how about details because of fire or terrorist attacks? By taking advantage of MIM, D2D communication can be very lucrative. If there are a lot of people using the device, multimedia real-time streaming will ensure quality of service. It is particularly vulnerable to DoS (Denial of Service) and should be considered unstable because of it. They can be avoided if the beam-forming antenna is used instead.

\section{CONCLUSION}

Gigabit MOD is a research project related to making the future 5G network even more successful It can also be applied to space communication in general. the main application of this new technology is to enhance data transfer rate and integrity Several MIMtask ideas are established in this document, including ones that can be tested in a prototype testing environment. Similar research can be conducted on space diversity and spatial multiplexing. What the experiment is looking for are different combinations of throughput and various spatial conditions for a throughput is being applied to. To make effective use of beam-forming and channel knowledge, plan the harvesting of particular energies will be done. It can be further developed fordeployment, MIM also.

\section{REFERENCES}

1. G. Wonder, P. Jung, M. Kasparick et al., "5G Non orthogonal, asynchronous wave form for future mobile applications," IEEE Communications Magazine, vol.52, no.2, pp.97-105, 2014.

2. J.G.Andrews, S.Buzzi, and W.Choi, "What Will 5G be? ", IEEE Journal on Selected Areas in Communications, v ol.32,no.6,pp.1065-1082,2014.

3. T.Rappaport, S.Sun, R.Mayzusetal ,“ Millimeter wave mobile communications for 5G cellular: it will work!," IEEE Access, vol.1,pp.335-349,2013.

4. E. G. Larsson, O. Edfors, F. Tufvesson, and T. L. Marzetta, "Massive MIMO for next generation wireless systems," IEEE CommunicationsMagazine, vol.52, no.2,pp.18 6-195,2014.

5. M. Kamel, W. Hamouda, and A. Youssef, "Ultra-dense networks: A survey" IEEE Communications. Surveys \& Tut orials,vol.18,no.4, pp.2522-2545, 2016

6. Jungnickel, V.,Manolakis, K.,Zirwas, W. et al.:"The role of small cells, coordinated multi point, and massive MIM $\mathrm{O}$ in 5G",IEEECommunicationsMagazine, 201 4,52,(5),pp.44-51.

7. Xu, J., Wang, J.,Zhu,Y. et al.: "Cooperative distributed optimization for the hyper-dense small cell deployment ",IEEE Communications Magazine, 2014, 52, (5):pp. 61-67.

8. Xu,J., Wang,J., Zhu, Y.etal.:"Cooperative distributed optimization for the hyper-dense small cell deployment ",IEEE Communications Magazine,2014, 52, (5):pp. 61-67.

9. T. Maksymyuk, M. Brych, I. Strykhalyuk, M. Jo, "Fractal Modeling for Multi-Tier Heterogeneous Networks with Ultra-High Capacity Demands," Smart Computing Review, vol. 5, no. 4, pp. 346-355, 
Aug.2015.

10. Liu, W., Han, S., Yang, C., Sun, C.:"Massive MIMO or small cell network: Who is more energy efficient?", Proc.of IEEE Wireless Communications and Networking Conference Workshops (WCNCW'2013), 2013, pp. 24-29.

11. Jo, M., Maksymy uk, T., Batista, R.L.et al.:"A Surve of Converging Solutions for Heterogeneous Mobile Networks', 2014, 21, (8), pp. 54-62.

12. Larsson, E., Tufvesson, F., Edfors, O., Marzetta, T. L."Massive MIMO for Next Generation Wire Systems ', IEEE Communications Magazine, 2013, 52, (2), pp. 186-195.

13. EmilBjörnson1,JakobHoydis2andLuca Sanguinetti3,"Massive MIMO Networks: Spectral, Energy, and Hardware Efficiency "University;emil.bjornson@ liu.se,BellLabs, Nokia;jakob.hoydis@nokia.com,University ofPisa;luca.sanguinetti@unipi.it

14. Konstantinos P. Liolis, Athanasios D. Panagopoulos, and Panayotis G.Cottis" Multi-Satellite MIMO Communications at Ku-Band and Above: Investigations on Spatial Multiplexing for Capacity Improvement and Selection Diversity for Interference Mitigation", Wireless\& Satellite Communications Group, School of Electrical and Computer Engineering, National Technical University of Athens(NTUA), 9 Iroon Polytech Street, Zografou,Athens 15780, Greece13May2007.

15. Samsung Electronics, "5GVision", White Paper, February 2015.

16. Karthik, A., MazherIqbal, J.L. Efficient Speech Enhancement Using Recurrent Convolution Encoder and Decoder. Wireless Pers Commun (2021). https://doi.org/10.1007/s11277-021-08313-6

17. Z. Gao, L. Dai, D. Mi, Z. Wang, M. A. Imran, and M. Z. Shakir, "Mm Wave Massive-MIMObased Wireless Backhaul for the 5G Ultra-Dense Network", IEEE

Wireless Communications,vol.22,no.5,pp.13-21,October 2015. 